
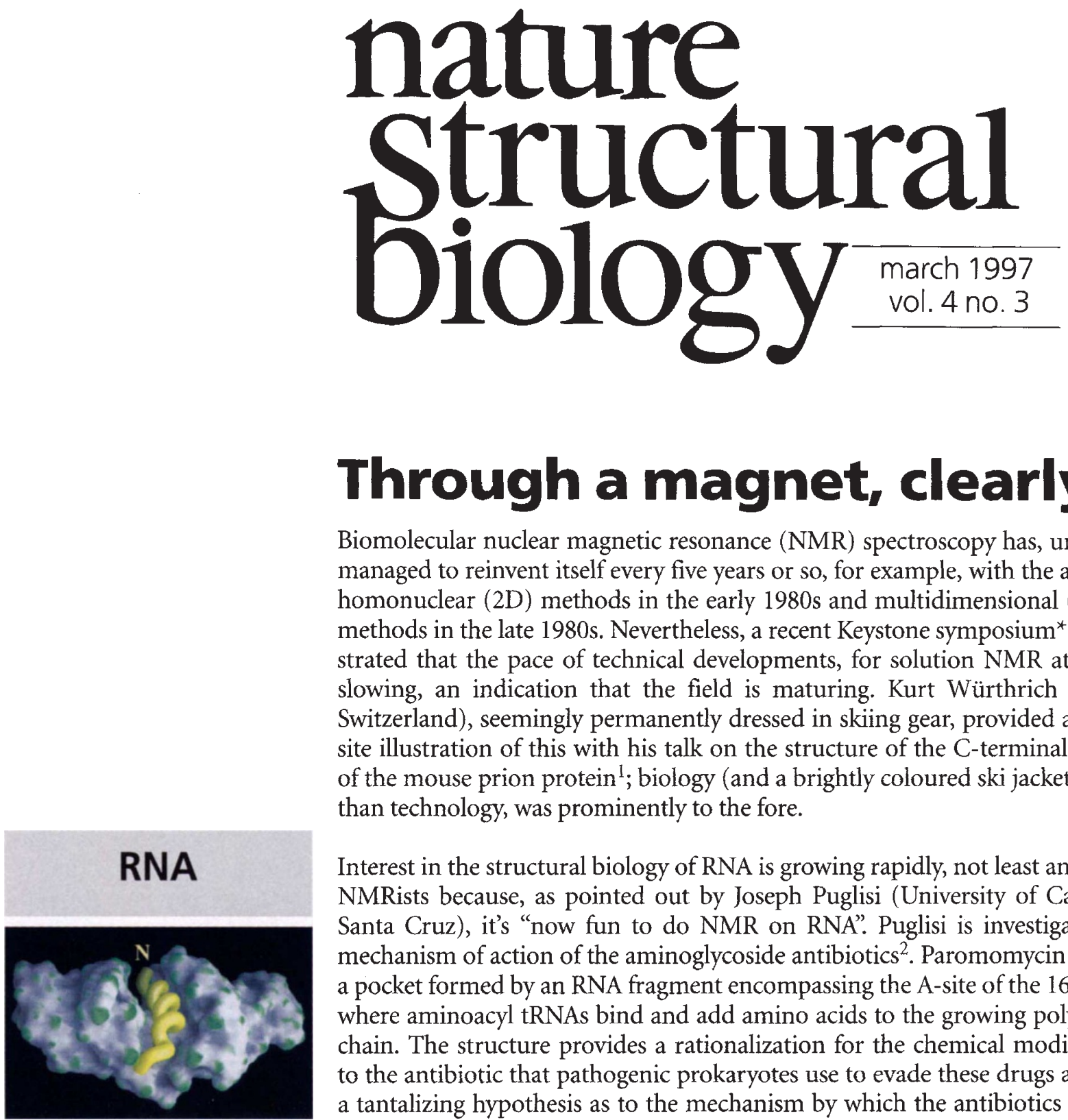

\title{
Through a magnet, clearly
}

Biomolecular nuclear magnetic resonance (NMR) spectroscopy has, until now, managed to reinvent itself every five years or so, for example, with the advent of homonuclear (2D) methods in the early 1980s and multidimensional (3D/4D) methods in the late 1980s. Nevertheless, a recent Keystone symposium ${ }^{\star}$ demonstrated that the pace of technical developments, for solution NMR at least, is slowing, an indication that the field is maturing. Kurt Würthrich (Zürich, Switzerland), seemingly permanently dressed in skiing gear, provided an apposite illustration of this with his talk on the structure of the C-terminal domain of the mouse prion protein ${ }^{1}$; biology (and a brightly coloured ski jacket), rather than technology, was prominently to the fore.

Interest in the structural biology of RNA is growing rapidly, not least among the NMRists because, as pointed out by Joseph Puglisi (University of California, Santa Cruz), it's "now fun to do NMR on RNA". Puglisi is investigating the mechanism of action of the aminoglycoside antibiotics ${ }^{2}$. Paromomycin binds in a pocket formed by an RNA fragment encompassing the A-site of the 16S rRNA, where aminoacyl tRNAs bind and add amino acids to the growing polypeptide chain. The structure provides a rationalization for the chemical modifications to the antibiotic that pathogenic prokaryotes use to evade these drugs as well as a tantalizing hypothesis as to the mechanism by which the antibiotics interfere with translation.

The structure of ATP-binding aptamers ${ }^{3,4}$ (Julie Feigon, Univ. California, Los Angeles; Dinshaw Patel, Sloan Kettering Cancer Center, New York) hint at the biochemical versatility of RNA and, perhaps, the RNA world, assuming you subscribe to this particular hypothesis ${ }^{5}$. Of course, in the post-RNA world RNAs function, for the most part, in conjunction with proteins, as illustrated by structures of the U1A protein-RNA hairpin ${ }^{6}$ (Gabriele Varani, MRC, Cambridge), Tat-TAR ${ }^{7,8}$ (Patel) and Rev-RRE $/$ aptamer ${ }^{10}$ (Jamie Williamson, Massachusetts Institute of Technology/Patel). The eIF-4E protein, which specifically binds to the 5'-cap structure in eukaryotic mRNAs and is part of the elF- $4 \mathrm{~F}$ complex involved in the initiation of translation, is under investigation by Gerhard Wagner (Harvard Medical School, Massachusetts). This unusually-shaped

${ }^{\star}$ Frontiers of NMR in Molecular Biology V, 6-11 February, Taos, New Mexico, USA molecule, consisting of an extended eight-stranded $\beta$-sheet and five $\alpha$-helices, binds cap analogues in a pocket formed by a series of loops on the upper edge of the protein. 


\section{editorial}

\section{Drug design}

\section{Membrane proteins}

\section{Technology}

1. Riek, R. etal. Nature 382, 180-182 (1996)

2. Fourmy, D., Recht, M.I., Blanchard, S.C. Puglisi, J.D. Science 274 1367-1371 (1996)

3. Jiang, F., Kumar, R.A., Jones, R.A. \& Patel, D.J. Nature 382, 183-186 (1996)

4. Dieckmann, T., Suzuki, E., Nakamura, G.K. \& Faigon, J. RNA 2, 628-640 (1996)

5. Miller, S.L. Nature Struct. Biol. 4, 167-169 (1997)

6. Allain, F.H-T, Gubser, C.C., Howe, P.W.A., Nagai, K., Neuhaus, D. \& Verani, G. Nature 380, 646-650 (1996)

7. Puglisi, J.D., Chen, L., Blanchard, S \& Frankel, A. Science 270 1200-1203 (1995)

8. Ye, X., Kumar, R.A. \& Patel, D.J. Chem. Biol. 2 , $827-840$ (1995)

9. Batiste, J.L. et al. Science 273, 1547-1551 (1996)

10. Ye, X., Gorin, A., Ellington, A.D. \& Patel D. Nature Struct. Biol. 3, 1026-1033 (1996)

11. Shuker, S.B., Hajduk, P.J., Meadows, R.P. \& Fesik, S.W. Science 274, 1531-1534 (1996)

12. Fraser, C. et al. Science 270, 397-403 (1995)
Perhaps the most striking demonstration of the utility of NMR as both a structural and a biochemical tool was 'structure activity relationships (SAR) by NMR' presented by Stephen Fesik (Abbot Laboratories, Illinois). The potential of the technology has been demonstrated for a model case, the FK506 binding protein ${ }^{11}$. Fesik now reported on the development of inhibitors against stromelysin, a matrix metalloproteinase implicated in a number of degenerative conditions, such as osteo- and rheumatoid arthritis, corneal ulceration, peridontal disease and degradation of the basement membrane in cancer metastasis.

Fesik uses NMR to screen for small molecules that interact with two separate sites on the surface of the protein and these are then optimized for binding resulting in $K_{\mathrm{D}} \mathrm{s}$ in the $\mathrm{mM}$ range, for example, $17 \mathrm{mM}$ and $0.02 \mathrm{mM}$ (high values that, initially, drew scorn from the medicinal chemists at Abbot Laboratories). Chemically linking these molecules together resulted in inhibitors with $K_{\mathrm{D}} \mathrm{s}$ in the $\mathrm{nM}$ range, a feat all-the-more impressive given that a regular screen of 115,000 compounds at Abbot yielded stromolysin inhibitors with $K_{\mathrm{D}}$ s no better that $10 \mathrm{mM}$. Furthermore, development of the high affinity leads using SAR by NMR took only six months.

One of the challenges for structural biology is the determination of the high resolution three-dimensional structures of membrane proteins. Stanley Opella (Univ. Pennsylvania, Philadelphia) observed that only a minority of biological functions are carried out by globular proteins and, further more, $30 \%$ of the genes of at least one organism, Mycoplasma genitalium (whose entire genome has been sequenced ${ }^{12}$ ), are putative membrane proteins.

NMR spectoscopy in aqueous solutions of mixed organic solvents is one trick being used the facilitate the structural analysis of membrane proteins, for example, the $12,000 M_{\mathrm{r}}$ multidrug transporter, EmrE (Manfred Schwaiger, Univ. Munchen, Germany). The use of micelles, which seem more faithful mimics of the cell membrane, allows 3D structure determination of membrane polypeptides of up to 120 residues (channel-forming peptides and Vpu from HIV-1, for example; Opella). But the size limit micelles impose on solution NMR experiments limits their utility. It is here that solid-state NMR should make a vital contribution to the field. Indeed, Opella reported the resolution of the solidstate NMR spectra for a 50 amino-acid membrane polypeptide in mechanically oriented bilayers; the structure solution should follow shortly.

As a number of speakers pointed out, an inherent limitation of NMR is the absence of restraints that define long-range interactions typical of macromolecules; NOEs can only provide information over a distance of $6 \AA$ or less. In an effort to correct this deficit Bax, Marius Clore and Angla Gronenborn (NIH, Bethesda) reported on the development and application of methods (magnetic susceptibility anisotropy and diffusion induced relaxation anisotropy) to derive true long-range NMR restrains. Equally important for eking structural information from NMR spectra are residue-, site- and atom-specific labelling protocols; for example, Williamson is developing enzymic methods for specially labelling nucleic acids.

The confidence and ability of the NMRists was clearly apparent in their conciliatory air towards the biological crystallography community, an indication that NMR is now quite capable of holding its own against the older, and presently more prevalent technology: in testament to this, the biology at the meeting (like the skiing) was quite stunning. 